\title{
I know how you feel: Task-irrelevant facial expressions are spontaneously processed at a semantic level
}

\author{
Stephanie D. Preston and R. Brent Stansfield \\ University of Michigan, Ann Arbor, Michigan
}

\begin{abstract}
Previous studies have demonstrated that emotions are automatically processed. Even with subliminal presentations, subjects involuntarily mimic specific facial expressions, are influenced by the valence of a preceding emotion during judgments, and exhibit slowed responses to personally meaningful emotions; these effects are due to reflexive mimicry, unconscious carryover of valence, and attentional capture, respectively. However, perception-action effects indicate that rapid processing should involve deep, semantic-level representations of emotion (e.g., "fear"), even in the absence of a clinical emotion disorder. To test this hypothesis, we developed an emotional Stroop task (Emostroop) in which subjects responded nonverbally to emotion words superimposed over task-irrelevant images of faces displaying congruent or incongruent emotional expressions. Subjects reliably responded more slowly to incongruent than to congruent stimuli, and this interference was related to trait measures of emotionality. Rapid processing of facial emotions spontaneously activates semantic, content-rich representations at the level of the specific emotion.
\end{abstract}

In social interactions, people infer others' emotions from their body posture, facial expressions, and movement, to respond appropriately (Darwin, 1872/1998). Facial expressions of basic emotions are recognized particularly accurately (e.g., Ekman, Sorenson, \& Friesen, 1969), and generating these expressions depends on distinct patterns of facial muscle activity (Ekman, Levenson, \& Friesen, 1983) that exist across cultures (Levenson, Ekman, Heider, \& Friesen, 1992) and are difficult to inhibit and falsify (Ekman, 2001). Thus, although somewhat under conscious control (Lanzetta, Cartwright-Smith, \& Kleck, 1976) and affected by experience in ontogeny (Ekman, 1973; Field, Pickens, Fox, Gonzalez, \& Nawrocki, 1998; Galati, Scherer, \& Ricci-Bitti, 1997), facial expressions of emotion are also innate and automatic (Izard, 1994).

Although consistent research on emotion processing indicates that emotions are processed automatically, generate a matching facial expression or emotional state, and influence subsequent judgments, none of these effects occur at an emotion-specific and semantic level across emotions and populations. Affective primacy, whereby subjects attribute subliminally presented emotions to subsequent neutral stimuli (Murphy \& Zajonc, 1993; Niedenthal, 1990; Stapel, Koomen, \& Ruys, 2002), does not occur when subjects consciously perceive the preceding face, occurs only at the level of valence, and is thought to result from processing that subverts cortical processing, going directly from the visual thalamus to the right amygdala (Bachmann \& Oit, 1992; Burton et al., 2003); however, it should be noted that amygdala activation to emotional faces can be attenuated by reduced attention, or particular subtractions (Pessoa, Japee, Sturman, \& Ungerleider, 2006; Pessoa, Padmala, \& Morland, 2005; Phillips et al., 2004). Facial mimicry, whereby people mimic facial expressions they perceive in others (Dimberg, Thunberg, \& Elmehed, 2000), does occur with subliminal and conscious processing of the face, is emotion specific, and can produce a matching state in the observer (cf. Buck, 1980); however, these effects are not thought to involve contextualized cortical representations - indeed, subjects can achieve emotional states from merely configuring their faces into expressions, without any knowledge of the emotion in the expression or task (Ekman et al., 1983). Mirror neuron studies of emotion do show cortical activation of the observer's feeling states in response to the target in both the insula (Carr, Iacoboni, Dubeau, Mazziotta, \& Lenzi, 2003; Singer et al., 2004) and somatosensory cortex (Adolphs, Damasio, Tranel, Cooper, \& Damasio, 2000), but do not involve more ventral representations of long-term memories and associations. In clinical studies on "intrusive cognition," clinical populations say (or read) the color of words more slowly if they are relevant to their disorder; these effects are emotion specific, but overly so, because they do not occur across emotions or in most nonclinical populations; instead, these effects appear due to an early attentional filter for perceiving stimuli that are either threatening or of particular survival relevance (e.g., food for a fasting individual; reviewed by Williams, Mathews, \& MacLeod, 1996). 
Combining across these literatures, there is good evidence for automatic processing of specific emotional information, which may produce a matching feeling state in the observer; however, none requires activation of longterm memory information related to the state, target, or situation and is demonstrable across specific emotions in the general population. Thus, current models of emotion processing cannot do justice to the following facts: (1) The lay definition of "empathy" requires the observer to have had an almost identical past experience; (2) most experiments on empathy find robust effects of familiarity and similarity (Preston \& de Waal, 2002); and (3) subjects with a similar past experience feel significantly more emotion than do those without such shared experience (Preston et al., 2007).

We theorize on the basis of the perception-action model (PAM; Preston \& de Waal, 2002) that when one observes the emotional state of a target, long-term semantic-level representations are activated rapidly and spontaneously, as information flows from cortical visual processing in the occipital lobe, moving anteriorly along the ventral stream in the temporal lobe including the fusiform gyrus, temporal pole, and hippocampus. Because this pathway proceeds from the slower, cortical visual route, these effects likely require conscious awareness of the target, but will nonetheless occur rapidly and without necessitating effort. This associative process activates the observer's own relevant memories, informing them as to the complexities and context of the other's state, to the extent that their experiences are similar.

To test this hypothesis, we used a novel emotional Stroop task (Emostroop) following the classic Stroop task (Stroop, 1935). Subjects selected the correct basic emotion category (happy, angry, sad) for the emotion word overlaid on a photograph of a congruent or incongruent emotional facial expression (happy, angry, sad, or neutral). If categorization of the emotion in faces is rapid, spontaneous, and at the level of the specific emotion, then the semantic encoding of incongruent faces should interfere with responses to the overlaid word, even within valence. Prior studies have also modified the classic Stroop task to create semantic interference between an emotional face and an overlaid word; however, each of these studies differs from ours in crucial ways.

In one study (Etkin, Egner, Peraza, Kandel, \& Hirsch, 2006), basic emotion terms were overlaid on emotional facial expressions, just as in our Experiment 1. However, in their study, subjects were instructed to attend and respond to the facial expressions, and the authors measured slowing from the task-irrelevant, incongruous words. This is akin to a true Stroop effect, demonstrating that words are processed automatically, which can affect processing in other domains. Because their subjects attended to the faces, they cannot measure the extent to which facial expressions are processed rapidly and spontaneously (only the extent to which words can interfere with the effortful processing of faces).

In another study (Haas, Omura, Constable, \& Canli, 2006), subjects were presented with positive, negative, or neutral words overlaid on positive, negative, or neutral faces. In this case, the subjects were indeed responding to the words (as in our task); however, the incongruence was measured only between valences, not across specific emotions within a valence. Thus, this study, like affective priming studies, is not able to measure the extent to which facial expressions of emotion access semantic representations of specific emotions.

In summary, both of these prior studies are like ours in the sense that they demonstrate semantic-level interference (unlike clinical attentional capture tasks), but none can test the extent to which specific facial expressions of emotion are processed rapidly and spontaneously. In order to fully understand emotion processing, and the mechanisms by which we can empathize with the emotions of others, we need to demonstrate that specific emotions are processed at a semantic level even when we don't specifically attend to them. This is the first study to demonstrate this fact.

\section{EXPERIMENT 1}

\section{Method}

Subjects. Fifty-three undergraduates at the University of Michigan participated in the experiment for course credit. All subjects completed the entire task and were included in the analysis ( 24 males and 29 females; mean age, 18.8 years; range, $18-21$ years). Fortyeight reported right-handedness and 5 reported left-handedness; all were without a history of psychiatric or neurological illness. The experiment was approved by the University of Michigan's Institutional Review Board, and all subjects gave informed consent in compliance with federal and institutional guidelines.

Stimuli. Subjects were tested individually on an iMac desktop computer in a single-subject testing room after receiving verbal instructions from the experimenter. Emostroop was programmed using Macromedia Flash (San Francisco) on a PC and was administered via Internet Explorer; this process did not introduce any noise to the collection of response time (RT) data during transfer to the server. Responses were made by all subjects using a mouse with a single-button click.

On each trial, the subject saw a blue cue-circle in the lower right corner of the screen. Clicking on the cue-circle caused it to disappear, and the stimulus and response buttons to appear simultaneously, starting the RT. Subjects clicked on one of the four response buttons "as quickly and accurately as possible," after which feedback was given in the form of a large green (correct) or red (incorrect) circle. Response buttons were quarter-circle arcs located at diagonals, radially around the location of the cue-circle, labeled as follows: HAPPY, SAD, SCARED, and ANGRY (Figure 1). Response button locations were randomized between subjects.

There were three blocks of trials: words only (32 trials), faces only (32 trials), and words and faces combined (64 trials). In the word-only block, the stimulus was one of the four emotion words (HAPPY, SAD, ANGRY, or SCARED, 8 trials each) in large, white, sansserif font, left of center on the screen; subjects responded by clicking the response button matching the word. In the face-only block, the stimulus was 1 of 16 Pictures of Facial Affect (PFA; Ekman \& Friesen, 1976), each appearing twice. The 16 pictures consisted of 1 picture displaying each of the four emotions (happy, sad, angry, and scared) from each of four actors (older man, younger man, blonde woman, and brunette woman), presented left of center on the screen; subjects responded by clicking the response button matching the facial expression. In the word-and-face block, the stimulus was 1 of the same 16 faces with one of the four emotion words superimposed semitransparently ( $89 \%$ transparent) over the face, centered vertically on the nose; subjects responded by clicking the response button that matched the word (Figure 1). On 48 trials in the word-and-face block, the facial expression matched the word (congruent trials-12 


\section{Experiment 1}

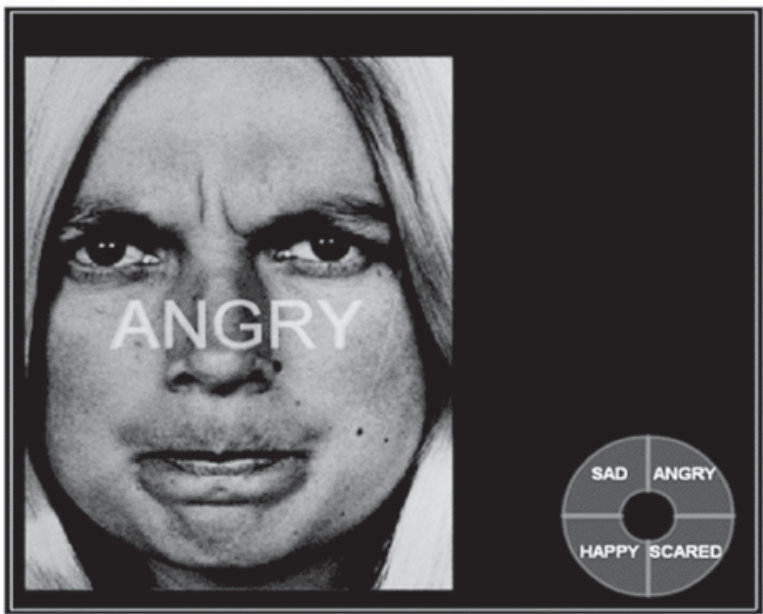

Experiment 2

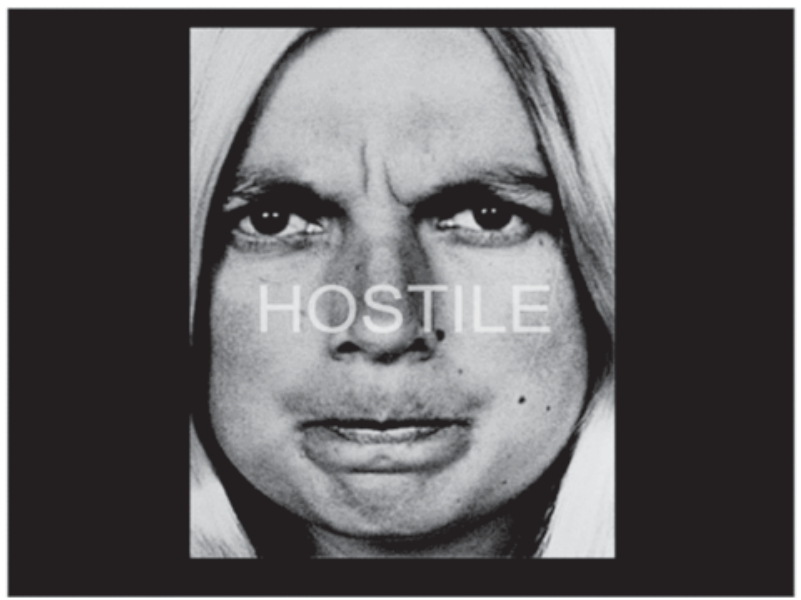

Figure 1. The combined word-and-face stimuli for Experiments 1 and 2. The first picture is a sample stimulus from Experiment 1 (administered via Flash animation using basic emotion words and mouse responses). The second picture is a sample stimulus from Experiment 2 (administered in E-Prime using prototypical emotion adjectives and keypress responses). Blocks 1 and 2 in Experiment 1 are not represented here, but consist of the same visual without the faces or without the words, respectively.

repetitions of each word matched to three iterations of each of the four actors expressing that emotion); on the other 16 trials, the facial expression did not match the word (incongruent trials - 4 repetitions of each word matched at random to one of the possible remaining incongruent facial expressions). The higher proportion of congruent trials was used in this experiment because Stroop effects are known to be sensitive to habituation; thus, for an initial, more liberal test of interference, unbalanced trial types were used to prevent habituation. The order of stimulus presentation was determined randomly for each block, for each subject.

Analysis. First, error rates were computed for each subject in each of the four conditions (word only, face only, congruent word and face, incongruent word and face), and errors were regressed by trial number to test for linear trends across Block 3 (separately for incongruent and congruent trials) to determine whether errors were related to task learning. Erroneous responses were excluded from further analysis.
Second, to test for the Emostroop effect, a paired, two-tailed $t$ test was used to compare median RTs (reducing sensitivity to the rightward skew of RTs) for each subject in congruent versus incongruent trials in Block 3 .

Third, a two-way ANCOVA was used to determine whether this Emostroop effect (difference between incongruent and congruent median RTs) differed by age, gender, or their interaction.

Fourth, in order to investigate possible differences in processing speed across the four stimulus types (words, faces, words and faces congruent, words and faces incongruent), a one-way repeated measures ANOVA was used, contrasting words with faces (to estimate differences in explicit stimulus processing), words with words and congruent faces (to estimate the RT benefit or cost of a dual stimulus), and words with words and incongruent faces (to estimate the interference effect).

Fifth, to check for RT differences among the specific stimuli, a general linear model (GLM) was run separately for Block 1 (words), Block 2 (faces), and Block 3 (words and faces combined), testing for main effects of word (four levels), actor (four levels), and facial expression (four levels), with subject identity entered as a random factor, reduced with iterative REML. Tukey honestly significant difference post hoc tests were used to compare levels of significant main effects. Although this GLM could be used to estimate the Emostroop effect if congruence were also entered as a factor, this contrast would not be fully factorial since incongruent pairings were determined randomly, resulting in missing data for multiple wordexpression combinations, making the power of such a comparison prohibitively low. Moreover, since each word has a unique congruent facial expression, congruence is nested within the word $\times$ expression interaction and is confounded with error variance from that interaction; the paired $t$ test is the more powerful and reliable test of the Emostroop effect.

All analyses were performed using JMP IN 5.1.2 for Mac OS $10.4, \alpha=.05$.

\section{Results}

Across all subjects, each button location held each emotion label fairly equally (ranging from $15 \%$ to $34 \%$ of subjects); these deviations did not differ from chance $\left[\chi^{2}(9)=13.1\right.$, n.s. $]$.

Average task time was $4 \mathrm{~min} 53 \mathrm{sec}(S D=37 \mathrm{sec})$. Fewer than half $(9 / 20)$ of the errors on incongruent trials matched the facial expression. A linear regression of number of errors $\times$ trial number (across all subjects) was not significant for incongruent trials $\left(R^{2}=.002\right.$, n.s. $)$ or for congruent trials $\left(R^{2}=.001\right.$, n.s.), suggesting that these errors were not due to unfamiliarity with the task or fatigue. All error trials and trials with RT greater than $3 S D$ s from the mean were eliminated for subsequent analysis.

Subjects took longer to respond on incongruent than on congruent trials [mean difference, $25.0 \mathrm{msec} ; t(52)=$ $3.15, p=.003, d=.45$; Figure 2]. This Emostroop effect did not differ by age, gender, handedness, or their interaction $[F(6,45)=0.96$, n.s. $]$.

RTs differed across the four conditions $[F(3,50)=$ $123.56, p<.0001]$ because subjects took less time to respond to words than to faces $[M=797.10$ and $1,200.10 \mathrm{msec}$, respectively; $F(1,52)=363.9, p<.0001]$ and to words with incongruent faces behind them $[M=$ $863.90 ; F(1,52)=27.67, p<.0001]$. Subjects also took less time to respond to words only than to words with congruent faces behind them $[M=838.90 ; F(1,52)=18.22$, $p<.0001$, indicating some processing cost for the combined stimuli. 


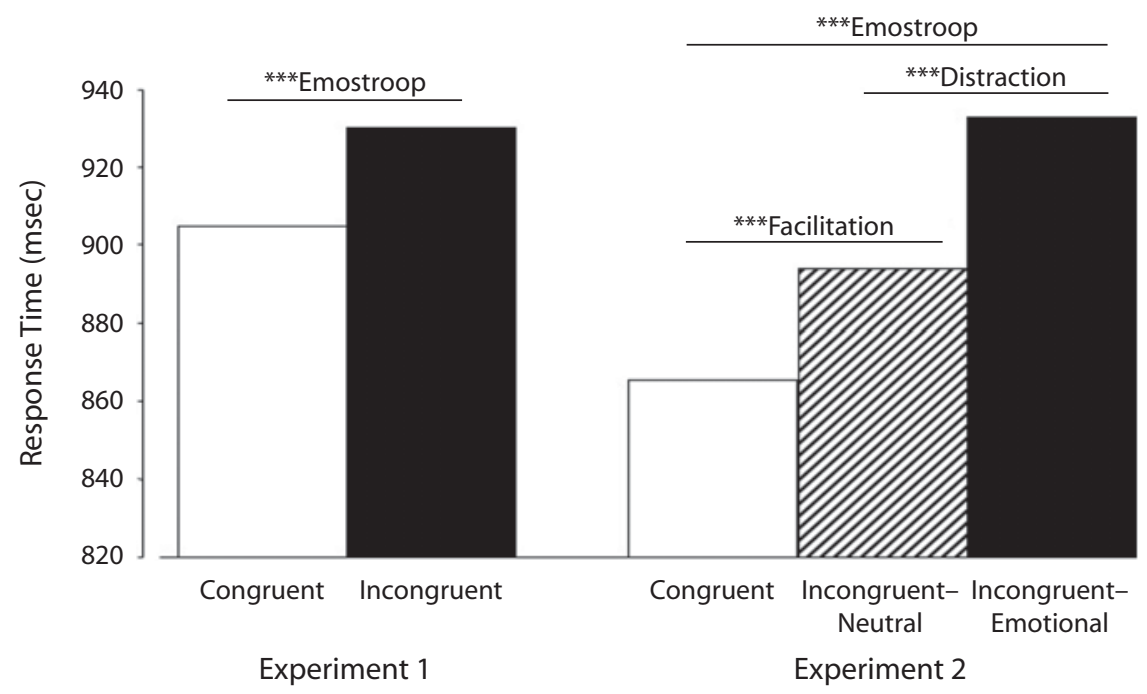

Figure 2. Response times across condition for Experiment 1 (on the left) and Experiment 2 (on the right) based on the mean of median response times (in milliseconds), averaged across subjects. Congruent trials are represented by unfilled bars, incongruent-neutral trials (Experiment 2 only) by hatched bars, and incongruent-emotion by filled bars. The Emostroop effect is the comparison of congruent- and incongruent-emotion trials in both experiments. In Experiment 2, the facilitation effect is the comparison of congruent- and incongruentneutral trials and the distraction effect is the comparison of incongruent-neutral and incongruent-emotion trials. ${ }^{* * *} p<.001$.

There were also significant differences among the individual stimulus items. In Block 1, there was a main effect of word $[F(3,1615)=5.83, p=.0006$; Table 1] due to the fact that responses were slower to ANGRY and SCARED than to SAD, with HAPPY intermediate.

In Block 2, there was a main effect of expression $[F(3,1440)=60.05, p<.0001$; Table 1$]$ due to the fact that responses to the facial expressions of anger were the slowest, significantly slower than to fear and happiness, whereas responses to expressions of happiness were the fastest, significantly faster than for all three negative emotions (anger, sadness, fear). There was a main effect of actor $[F(3,3159)=3.97, p=.008]$ due to the fact that responses to the older man were slower than to the brunette woman, with the other two actors intermediate. In a post hoc gender contrast between the two female actors and the two male actors, RT was significantly influenced by the gender of the actor $[F(1,1440)=5.44, p=.02]$, with responses slower to males than to females.

In Block 3, responses to the word ANGRY were significantly slower than to the word SAD, with SCARED and HAPPY intermediate [main effect, $F(3,3159)=28.07, p<.0001$; Table 1]. There were no differences in responses due to actor or facial expression $[F(3,3279)<1.36$, n.s. $]$.

\section{Discussion}

Subjects in Experiment 1 responded more slowly when the task-irrelevant background facial emotion was incongruent to the overlaid word than when it was congruent. This suggests spontaneous and rapid activation of semantic-level representations for emotions from facial expressions.

The effect is not likely due to confusion about the instructions (i.e., subjects responding to the face in Block 3), since errors were not related to trial number, the incongruent errors usually did not match the background face, and all error responses were removed from further analyses. Subjects did take significantly longer to respond when even a congruent face was added to the stimulus, suggesting that the mere presence of a face may have introduced some attentional cost in processing the word, similar to the attentional capture effect in clinical populations (Williams et al., 1996). The small size of our effect is consistent with its being a reverse Stroop effect, because word processing is thought to be harder to disrupt than visuospatial processing (Algom, Chajut, \& Lev, 2004).

There were differences in RT across the four actors, with responses to males, especially the older male, being

Table 1

Median Response Times (in Milliseconds) Averaged Across Subjects for Each Emotion, in Each Block, for Experiment 1

\begin{tabular}{ccccc}
\hline Block & Stimulus & Respond & Emotion & Response Time \\
\hline 1 & Word only & Word & ANGRY & $883.42^{\mathrm{a}}$ \\
& & & SCARED & $870.09^{\mathrm{a}}$ \\
& & HAPPY & $834.70^{\mathrm{a}, \mathrm{b}}$ \\
\multirow{3}{*}{2} & \multirow{3}{*}{ Face only } & Face & SAD & $812.34^{\mathrm{b}}$ \\
& & ANGRY & $1,586.08^{\mathrm{a}}$ \\
& & SCARED & $1,377.93^{\mathrm{b}}$ \\
& & & HAPPY & $1,058.97^{\mathrm{c}}$ \\
3 & \multirow{3}{*}{ Word and face } & \multirow{2}{*}{ Word } & SAD & $1,484.20^{\mathrm{a}, \mathrm{b}}$ \\
& & & ANGKY & $955.75^{\mathrm{a}}$ \\
& & SCARED & $945.43^{\mathrm{a}, \mathrm{b}}$ \\
& & & HAPPY & $850.00^{\mathrm{a}, \mathrm{b}}$ \\
& & & SAD & $835.25^{\mathrm{b}}$ \\
\hline
\end{tabular}

Note-Superscripts $(a, b)$ are based on Tukey post hoc tests to indicate significantly different emotion conditions within each block and experiment. 
slower than to the females, especially the brunette woman. This effect is consistent with previous research on recognizing facial expressions of emotion, which has found faster responses to females than to males (e.g., Palermo \& Coltheart, 2004).

There were differences in the RT to the four emotion words. Anger may be a more difficult emotion to categorize, because a prior study similarly found less accuracy for angry expressions than for happy and sad ones (Hess, Blairy, \& Kleck, 1997), and many experiments find faster and more accurate responses for happy expressions (e.g., Feyereisen, Verbeke-Dewitte, \& Seron, 1986). However, anger was also slower to be categorized in word-only trials. This could be due to differences in action tendencies associated with different emotions, because anger may adaptively prime individuals to cease action (Winkielman, Berridge, \& Wilbarger, 2005), or it may be due to attentional capture, which may be stronger for anger (e.g., McKenna $\&$ Sharma, 1995). It is also possible that differences among emotion words are simply due to a superficial characteristic in the stimuli, such as word length (e.g., SAD is noticeably shorter than the other three words and subjects may use that superficial characteristic to aid response selection).

Although the Emostroop effect was significant, a few aspects of the present design cast doubt on the interpretation of the effect as being due to rapid, spontaneous semantic-level processing of the emotional faces. The three-block format of Experiment 1 mirrored the original Stroop task and provided subjects with practice understanding the emotion in the photographs. However, attending to the faces in Block 2 may have biased subjects to attend to the irrelevant faces in Block 3. Also, the uneven sampling of congruent and incongruent trials in Block 3 may have biased subjects to attend to the faces since, in the majority of cases, the facial expressions were informative about the correct response. In addition, our interpretation of the effect as occurring at a semantic level is weakened by the response format (mouse movements to the onscreen response wheel) because subjects could use a templatematching strategy to match the word with the response, without having to encode the meaning of the word. These mouse movements also introduced RT noise that may have reduced the size of the Emostroop effect. There were also tests we could not run with the present design, such as a strong test of emotion-specific effects (given the uneven, random sampling of trials) and a test of interference and facilitation (given the lack of a neutral face). Thus, a more rigorous test of the Emostroop effect was required.

Experiment 2 addressed these concerns. The first two blocks were eliminated, the number of congruent and incongruent trials was equalized, a fully factorial design with equal numbers of trials in each actor-word-expression combination was used, neutral faces replaced fear faces, and response keys labeled only with the first letter of each word and covered by subjects' fingers were used for the response. Fear was eliminated as an emotion, to make the nonvisual response mapping easy to learn; within-valence tests of the Emostroop effect can still be done using anger and sadness. In addition, Experiment 2 was designed to demonstrate the semantic level of the effect by present- ing synonyms of the basic emotions on the screen and asking subjects to categorize each adjective into one of the three basic emotion categories (happy, sad, angry). This made the task and thus the nature of the Emostroop interference especially semantic in nature, eliminating template matching as a possible strategy and eliminating word length as a possible explanation for differences in RT across emotions.

\section{EXPERIMENT 2}

\section{Method}

Subjects. Sixty-three undergraduates at the University of Michigan participated for course credit (30 males and 33 females; mean age, 18.8 years; range, 18-22 years). Fifty-nine reported righthandedness and 4 reported left-handedness; all were without a history of psychiatric or neurological illness. The experiment was approved by the University of Michigan's Institutional Review Board, and all subjects gave informed consent in compliance with federal and institutional guidelines.

Stimuli. Subjects were tested individually on a Dell PC desktop computer in a single-subject testing room after receiving verbal instructions from the experimenter. The task and analyses were identical to those used in Experiment 1, with the following exceptions: The experiment was administered using E-Prime Version 1.1 experiment-presentation software (Psychology Software Tools, Inc., Pittsburgh, PA). All trials were word-and-face combined. All 384 combinations of words and faces were created in advance using Adobe Photoshop (Adobe Systems Inc., San Jose, CA). Words were overlaid on the PFA pictures, centered horizontally within the frame and centered vertically at the level of the vertical midpoint of the nose. Arial font, point size 46, in all capital letters, was used with $25 \%$ opacity (Figure 1 ).

Subjects were asked to respond to the word "as quickly and accurately as possible." Subjects responded to the word by pressing a corresponding key on the keyboard. Three emotion responses were possible: HAPPY, SAD, and ANGRY. Subjects responded using the index, middle, and ring fingers of their dominant hand on three keyboard keys labeled with an $H, S$, and $A$ sticker (respectively; order randomized across subjects).

On each trial, the subject saw a black screen for $500 \mathrm{msec}$ before seeing the word-and-face stimulus. The stimulus remained on the screen until the subject responded. Feedback was given after each response in the form of a colored border around the picture (green for correct, red for incorrect) for $500 \mathrm{msec}$ before the black screen came up again to start the next trial.

The overlaid words were taken from a list of eight emotion adjectives determined to be prototypical of the three basic emotion categories in a prior investigation (Shaver, Schwartz, Kirson, \& O'Connor, 1987). Happy was represented by blissful, cheerful, gleeful, jolly, jovial, joyful, delighted, and glad. Sad was represented by depressed, hopeless, gloomy, glum, grieving, sorrowful, woeful, and miserable. Angry was represented by enraged, outraged, furious, wrathful, hostile, bitter, hateful, and scornful.

Each facial expression was represented an equal number of times by each actor in each condition. On each trial, one of the eight words for the response category was selected at random. The stimuli for the congruent trials consisted of 96 pictures ( 1 for each emotional expression, for each of the four actors, with each of the possible eight corresponding emotion adjectives overlaid). The stimuli for the incongruent-emotion trials consisted of 192 pictures (each emotional expression was paired with all of the eight emotion adjectives from the other two emotions, for each of the four actors). The stimuli for the incongruent-neutral trials consisted of 96 pictures (each of the eight emotion adjectives for the three emotion categories was paired with a neutral expression behind it, for each of the four actors). To produce the same number of congruent and incongruent trials despite different numbers 
of possible combinations in each condition, emotion adjectives were selected at random within the pool of possible emotion adjectives for that emotion category. Therefore, for each subject, each of the 96 possible congruent pictures was shown twice, producing 192 congruent trials; only half of the 192 possible incongruentemotion pictures were shown, producing 96 incongruent-emotion trials, and all 96 of the incongruent-neutral trials were shown, for a total of 384 trials, which were randomly selected without replacement by the program, separately for each subject.

After all Emostroop trials were complete, subjects completed the Doherty Emotional Contagion Scale (Doherty, 1997), hereafter referred to as EC, which produces a total EC score, as well as separate subscale scores for the tendency to resonate with specific emotions, including happy, love, fear, angry, and sad. Subjects also completed the Beck Depression Inventory-II (BDI-II; Beck, Steer, \& Garbin, 1988).

Analysis. Analyses were identical in form to those of Experiment 1 , with the following exceptions. The second analysis, testing for the Emostroop effect, was changed from a $t$ test to a one-way ANOVA, to accommodate the incongruent-neutral condition, with two-tailed paired $t$ tests determining post hoc which conditions differed significantly. The fourth analysis in Experiment 1 concerned the comparison of word-only and face-only conditions with wordand-face conditions; since all trials in Experiment 2 contained words and faces, that analysis was not performed.

An additional analysis was done for Experiment 2 to further rule out an alternative hypothesis that the Emostroop effect is not emotion specific, but is driven by the opposition between positive and negative stimuli (e.g., happy paired with either sad or angry), and not by stimuli that differ at the level of the specific emotion, within a valence (e.g., sad paired with angry). To test for this, we compared RTs on three types of incongruent trials: (1) nonerror [ $Z(\mathrm{RT}) \leq 3.0]$ trials with a happy word and a sad or angry expression, (2) trials with a sad or angry word and a happy expression, and (3) trials with a sad word and an angry expression or vice versa. If the Emostroop effect is due to an opposing valence, then RTs to Types 1 and 2, where the stimuli are of opposing valence, should be significantly longer than RTs to Type 3, where both are negatively valenced. A GLM of RT with subject identity entered as a random factor modeled RT by these three types.

Given that we now have a fully balanced design, we can also examine more closely the emotion-specific Emostroop effects. Each emotion-specific Emostroop score represented the RT cost incurred from having that facial expression behind each possible incongruent emotion word, compared with what the RT was when the word had a congruent expression behind it (e.g., the median for when a sad word has a happy face behind it minus the median for when a sad word has a sad face behind it). We used the median RTs for each word and face pairing (12 pairings, based on three emotion word categories and four facial expressions, including neutral) and used them to create difference scores between the congruent and incongruent pairings. There are two difference scores for each of the emotion faces (one pairing for each of the other two emotions) and three difference scores for neutral (because it is incongruent with all three emotion words). These difference scores were averaged together (within each expression) and used as the emotion-specific Emostroop effect for that subject.

A one-way, repeated measures ANOVA compared the size of the four emotion-specific Emostroop effects. Intercorrelations of the effects, and their correlations with the personality measures, were computed.

All analyses were performed using JMP IN 5.1.2 for Mac OS $10.4, \alpha=.05$.

\section{Results}

Each session lasted approximately $30 \mathrm{~min}$. Error rates were similar across conditions. On incongruent trials, more $(64.6 \%)$ of the erroneous responses matched the facial expression than did not, justifying the exclusion of these trials from the analysis.
The words generated their appropriate responses overwhelmingly. Some words generated more errors than did others. BITTER and SCORNFUL (prototypical anger words) generated many "sad" responses $(13.95 \%$ and $7.29 \%$, respectively), whereas MISERABLE (a prototypical sad word) generated many "angry" responses (7.36\%). These effects were considered noise; trials on which subjects correctly categorized these words were included in subsequent analyses. The linear regression of number of errors by trial number (across all subjects) was significant for incongruent trials $\left(R^{2}=.0015, p<.0001\right)$ and for congruent trials $\left(R^{2}=.0014, p<.005\right)$, but not for neutral trials $\left(R^{2}=\right.$ .0004, n.s.). All effects were very small but positive, suggesting that errors were likely due to minor fatigue. All error trials and trials with RT greater than $3 S D$ s from the mean were eliminated for subsequent analysis.

RTs differed significantly across the three conditions [congruent, incongruent-emotion, and incongruentneutral, $F(2,61)=35.87, p<.0001$; Figure 2]. Incongruentemotion RTs were on average $67.82 \mathrm{msec}$ longer than congruent RTs [paired $t(62)=8.50, p<.0001$ ]; this Emostroop effect did not differ by age, gender, or their interaction $[F(3,58)=0.949$, n.s.]. Congruent responses were on average $28.63 \mathrm{msec}$ shorter than incongruent-neutral responses [paired $t(62)=3.82, p<.001$ ]; this facilitation effect did not differ by age, gender, or their interaction $[F(3,58)=0.458$, n.s. $]$. Incongruent-emotion RTs were on average $39.19 \mathrm{msec}$ longer than incongruent-neutral RTs [paired $t(62)=5.23, p<.0001$ ]; this distraction effect did differ significantly across subjects $[F(3,58)=2.787, p<$ $.05]$ due to an age $\times$ gender interaction $[F(1,58)=7.838$, $p<.01]$ only because the 2 oldest subjects were male, and showed negative cost, whereas the 2 oldest females had high cost. Removal of these 4 subjects reduced the effect to insignificance $[F(1,54)=0.103$, n.s. $]$.

RTs across subjects in the GLM were heavily skewed (skewness $=6.57$ ). Eliminating error trials and ones with RTs that were at least $3.0 \mathrm{SD}$ s greater than the mean resulted in removal of 385 trials out of 24,192 trials total (1.59\%), across all 63 subjects (from 3,256 msec to $24 \mathrm{sec}$ long). Responses again differed across the specific stimuli (Figure 3). There was a significant effect of word emotion $[F(2,22422)=128.09, p<.0001]$, with responses to sad and angry words again taking longest $(M=1,068.95$ and $1,063.28 \mathrm{msec}$, respectively), significantly longer than those to happy words $(M=953.79 \mathrm{msec})$. There was a main effect of facial expression $[F(3,22422)=5.37, p<$ .005], with RTs on trials with happy expressions taking longer $(M=1,050.27 \mathrm{msec})$ than trials with angry or neutral expressions $(M=1,024.65$ and $1,014.34 \mathrm{msec}$, respectively). The differences between these two comparisons (happy words faster but happy faces slower) also produced a word emotion $\times$ expression interaction $[F(6,22422)=$ $21.30, p<.0001]$. Finally, there was an interaction of actor $\times$ expression $\times$ word emotion $[F(18,22422)=1.85$, $p<.025$ ] due mainly to the large Emostroop effect on happy word trials involving one of the male actors.

The opposing-valence test found a significant difference between the three types of incongruent trials $[F(2,39)=$ $25.590, p<.0001]$, but not in a pattern predicted by an 


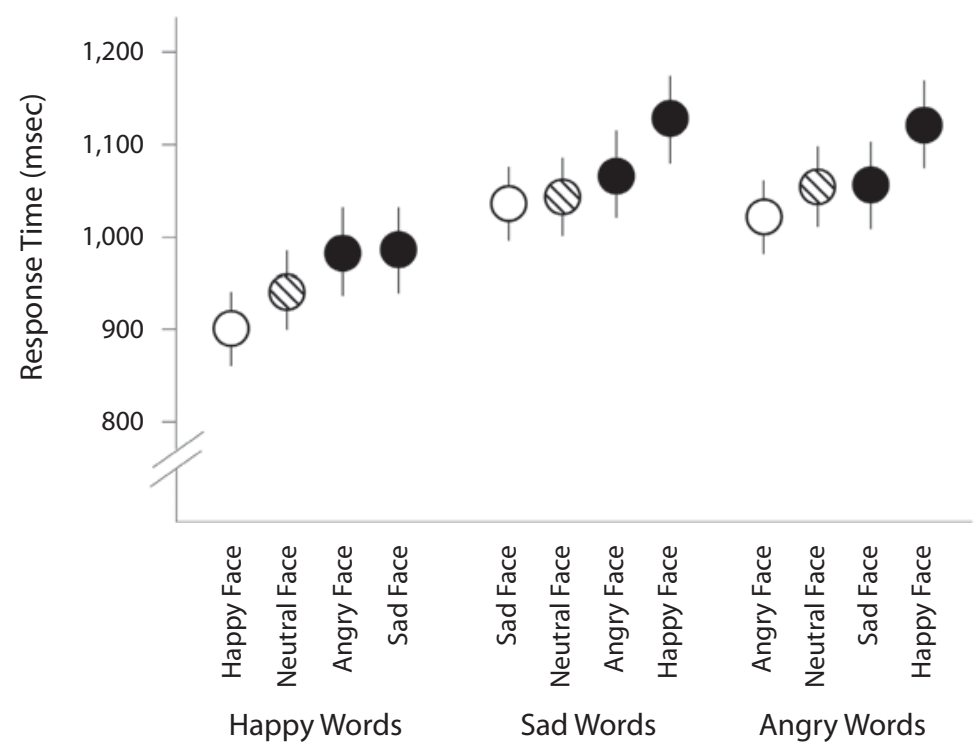

Figure 3. Response times (least squares means, $95 \%$ confidence intervals) from the word $\times$ expression interaction in Experiment 2 . Congruent trials are represented by unfilled circles, incongruent-neutral by hatched circles, incongruent-emotion by filled circles. Trials are grouped by emotion word, with the facial expression labeled vertically on the $x$-axis.

opposing-valence hypothesis since the within-valence trials were not the fastest. RTs to negative words with happy faces $(M=1,125.03 \mathrm{msec})$ were significantly longer than RTs to negative words with negative faces $(M=1,065.33 \mathrm{msec})$, which were significantly longer than RTs to the positive word with a negative face $(M=993.83 \mathrm{msec})$.

The size of the four emotion-specific Emostroop effects differed significantly $[F(3,60)=14.551, p<.0001]$, with happy being the most distracting, angry and sad the next most distracting, and neutral the least distracting. The four scores were all positively correlated, although not significantly (although there were trends with $p<.10$ for the positive correlation of neutral and happy and of angry and sad).

The overall and emotion-specific Emostroop effects show some meaningful correlations with the collected scale data (Table 2). The overall Emostroop effect had a trend toward being larger in subjects with higher levels of depression on the BDI-II. Similarly for the emotion-specific Emostroop effects, subjects who were more depressed had significantly larger neutral Emostroop effects (which is usually the smallest Emostroop effect) and a trend toward larger anger Emostroop effects; this means that subjects with higher levels of depression were more sensitive to neutral faces, and somewhat to angry faces. The BDI-II had a mean of $7.67(S D=5.60)$, within the range of normal scores, but the range was $0-27$, with two subjects falling in the depressed range (21 and 27). Subjects with high happy Emostroop effects showed significantly lower emotional contagion for anger, and a trend toward lower emotional contagion in general, and lower contagion of fear.

\section{Discussion}

The Emostroop effect was again reliable, even after controlling for possible confounds and testing for the role of valence incongruity. Most importantly, in this experiment, subjects were not trained to categorize faces (as they were previously in Block 2), and there was no benefit to attending to the face (as there was previously in Block 3 ). Thus, the presence of the emotional faces was truly task irrelevant and could not benefit performance, yet subjects still processed them spontaneously, resulting in slowed performance when the face did not match the word to which they were supposed to respond.

RTs to trials with a congruent background expression were faster than those with a neutral background expression, evidence for facilitation and additional evidence that the emotion of the task-irrelevant, background faces affects responses to the word.

There was again an effect of actor, and again the older man produced the slowest responses, whereas responses to the younger man were significantly shorter. Taking

Table 2

Experiment 2 Pearson Correlations ( $r$ ) of Emotion-Specific Emostroop Effects With Emotional Trait Scales

\begin{tabular}{lcrrrl}
\hline & \multicolumn{5}{c}{ Emotion-Specific Emostroop Effects } \\
\cline { 2 - 6 } & Happy & Angry & Sad & Neutral & Overall \\
\hline EC total & $-.215^{+}$ & -.022 & .034 & -.006 & -.079 \\
Happy & -.171 & .004 & .142 & .034 & -.082 \\
Love & .012 & .027 & -.124 & -.088 & -.032 \\
Fear & $-.233^{+}$ & -.096 & .144 & -.041 & -.032 \\
Anger & $-.298^{* *}$ & .011 & -.067 & -.043 & -.074 \\
Sad & -.101 & -.008 & .049 & .111 & -.070 \\
BDI-II & .029 & $.215^{+}$ & -.069 & $.311^{* *}$ & $.214^{+}$ \\
\hline
\end{tabular}

Note-The emotion-specific Emostroop effects refer to the degree to which response times to that emotion word were slowed by the presence of an incongruent facial expression, compared with a congruent facial expression. ${ }^{+} p<.10 .{ }^{* *} p<.025$. 
these data together with the first experiment, age is most explanatory, perhaps in keeping with studies of automaticity that find slowed walking after priming of elderly stereotypes (Bargh, Chen, \& Burrows, 1996). Notably, both male actors appear as if they could be older than both female actors; thus, the fastest responses to the brunette woman and the slowest responses to the older man (in the previous experiments) could be solely related to the age of the actors, or some correlated variable such as youthfulness or fecundity.

Whereas the first experiment used a matching task, this experiment used a semantic categorization task that enhanced most of the Emostroop-related effects. The previous Emostroop effect was reliable, but small (approximately $25 \mathrm{msec}$ ); in this experiment, the Emostroop effect was $68 \mathrm{msec}$, almost three times larger, which is expected given that the increased cognitive demand of categorizing produces more response variability, which increases the potential for interactions.

There was again a main effect of the emotion of the word. RTs to anger words were very slow, whereas RTs to happy words were very fast. RTs to sad words were similar to those to anger, significantly longer than those to happy words; this supports the supposition above that the faster RTs to SAD in Experiment 1 were due to the short word length, which made it easier to recognize.

In contrast, this is the first time that RTs to happy facial expressions were slower. The most parsimonious explanation is that the emotion word effects are very strong and are exacerbated when subjects make categorical distinctions; thus, incongruent trials with happy faces behind them seemed slow, but they might be better thought of as trials on which negative words were being semantically processed.

The separate valence-opposition test did not find slower RTs for the two opposing-valence combinations than for the within-valance combination. Although there may be effects of valence incongruity, they do not account for the Emostroop effect.

There were some interesting relationships between the Emostroop effects and self-report data. Individual differences in emotional contagion seemed particularly related to subjects' tendency to be distracted by a happy face-people with high levels of emotional contagion, especially for anger, but also somewhat overall, and for fear, were less distracted by a happy face. This could be because people who can relate strongly to negative emotions are less empathic for positive emotions. It could also be because people who relate strongly to negative emotions are particularly responsive to the negative emotion words, making them less susceptible to interference from happy. It is also conceivable that people who are less sensitive to positive emotions in others end up either feeling more negative affect, or being more likely to perceive it in others. Similarly, there were relationships between the Emostroop effect and the BDI-II, even though we used a population prescreened to exclude individuals with depression. People with higher levels of depression tended to have larger Emostroop effects overall, had significantly larger Emostroop effects for neutral faces, and showed a trend toward having a larger Emostroop effect for anger. This is especially striking since neutral faces produced the lowest Emostroop effect overall. Given research that shows that people can interpret neutral facial expressions as angry, especially early in development (Thomas et al., 2001), this result may support the idea that people with depression are actually more sensitive to anger or require more positive reinforcement, and thus interpret neutral faces as angry, or assume anger in the absence of normal, positive social cues. Future research should investigate the Emostroop effect in depressed populations, taking care to separate responses to incongruence per se from tendencies to attend to particular negative emotions.

These findings suggest that Emostroop is a domaingeneral effect that occurs across people and emotions, but is also influenced by individual tendencies regarding specific emotions (like the clinical intrusive cognition experiments). It is important to emphasize that the Emostroop effect is not due to the same processing cost as intrusive cognition effects. Although the intrusive cognition effects are due to distraction from the personally relevant emotional cues, the Emostroop effect is due to semantic-level interference or response competition between the representation for the word and the face. Because of this basic property, our effect is reliable across all emotions, including happy, sad, fear, anger, and neutral, and in populations that are prescreened to be without a history of psychiatric illness.

\section{GENERAL DISCUSSION}

The presence of the original color-naming Stroop effect suggests that reading occurs rapidly and spontaneously, is difficult to inhibit, and occurs at a semantic level. The data presented here suggest that the same is true of the perception and understanding of others' emotional facial expressions. Even when the emotional faces did not predict the overlaid word, and with RTs well under a second, it took subjects as much as $70 \mathrm{msec}$ longer to respond to words with incongruent background faces than to congruent ones. This Emostroop effect suggests that facial expressions are not only automatically mimicked, or processed at the level of valence, but also semantically understood at relatively short intervals.

The size of the original, color-naming Stroop effect varies by individuals, by stimuli and task design, and by the amount of experience subjects have with the task, suggesting that reading color words is not out of conscious control, just that it is normally processed without effort, and even requires effort to not process; this variability is even considered a necessary hallmark of any true Stroop-like effect (Algom et al., 2004). Similarly, we would expect that whereas emotion in facial expressions is normally processed without conscious effort, it does differ across individuals and can be controlled with effort or practice.

Stroop effects are typically asymmetrical, with incongruent words slowing performance on the ink-colornaming task more than incongruent ink colors slow performance on word reading. Emostroop resembles the latter, which MacLeod (1991) calls a "reverse Stroop effect," and which has a smaller effect size than the classic effect. The 
reliability of the effect is noteworthy given the difficulty of interfering with such a well-honed skill as word reading.

Moreover, this is a highly replicable effect, which we have demonstrated in six independent samples (four not presented here), using both nouns and verbs, and even after allowing subjects twice as much practice, or as many as 240 incongruent trials (Preston \& Stansfield, 2006, 2007).

The facilitation effect suggests that congruent faces are not distracting, but actually speed responding - more evidence that they are processed and understood rapidly. Similar to the "intrusive cognition" effect documented in clinical populations (McKenna \& Sharma, 1995), there was also a distraction effect, with emotional facial expressions slowing responses compared with neutral expressions.

RTs were consistently slower to angry words and faster to happy words. The fact that this effect occurred even in the absence of faces (in Block 1 of Experiment 1) suggests that the effect is not due to stimulus properties of facial expressions (e.g., anger being harder to detect) but results from the inherent, conditioned social response to detecting anger, which likely suppresses behavior in the service of gathering more information, or to avoid exacerbating the situation (e.g., Hommel, 2003; Schwarz \& Clore, 1996; Winkielman et al., 2005).

Importantly, although we did find the previously reported effects of action affordance, distraction from emotional faces, and effects of valence, the effect of slowed processing due to an incongruent background expression remained even after partialling out the former effects.

Unlike affective priming (Murphy \& Zajonc, 1993) or motor mimicry (Dimberg et al., 2000) effects that can occur with subliminal presentation of the faces, we do not expect the Emostroop effect to occur without conscious processing of the face since it should rely on processing that occurs via cortical, occipital processing, proceeding through visual association areas and on to representations in the ventral stream, likely even reaching as far as the left inferior frontal gyrus. This level of processing would necessarily take longer than mechanisms that only preprocess the stimuli, but they also allow subjects to appraise the other's state and respond appropriately, a necessary requirement for empathy.

According to a PAM of empathy (Preston \& de Waal, 2002), people understand the emotion of others by activating their own representations for the state, including facial expressions, body postures, related past experiences, and appropriate responses. Other models already suggest and find evidence for automatic mimicry of facial expressions (e.g., Dimberg et al., 2000), activation of one's own motor representations from observation of another's motor act (e.g., Grafton, Fadiga, Arbib, \& Rizzolatti, 1997), and even activation of the substrates of feeling from observing others' basic emotion (Carr et al., 2003; Decety \& Chaminade, 2003; Wicker, Perrett, Baron-Cohen, \& Decety, 2003) or pain (Singer et al., 2004). However, other models do not require, and have not looked for, spontaneous activation of semantic-level information from mere perception, especially in a context in which the task did not make explicit reference to or require elaborated pro- cessing of the facial expression. This semantic-level, conceptually rich information, which is distributed throughout the ventral processing stream, including the inferior temporal lobe, temporal pole, and hippocampus, allows one to truly understand the emotional state of another, obligatorily, to the extent that they have a matching representation for that state.

This task can be useful for basic research into the level of impairment in individuals with emotion-processing deficits. For example, individuals with frontal lobe damage, psychopathy, and autism show reduced empathy for the feelings of others, but the exact level of their impairment is poorly understood and thought to differ. Eyetracking could be added to even better interpret null effects.

Because the Emostroop task reliably produced interference from task-irrelevant facial expressions and still found emotion-specific effects consistent with the literature, it could also be used to investigate processing differences for specific emotions. For example, additional emotions can be added as necessary to test for emotion-specific impairments, as has been suggested, but debated, for schizophrenia (e.g., Archer, Hay, \& Young, 1994; Schneider et al., 2006; Silver, Shlomo, Turner, \& Gur, 2002), autism (Baron-Cohen et al., 1994; Hobson, Ouston, \& Lee, 1989; Kamio, Wolf, \& Fein, 2006), Huntington's disease (Gray, Young, Barker, Curtis, \& Gibson, 1997; Sprengelmeyer et al., 1996; Sprengelmeyer et al., 1997), and developmental disturbances (de Wied, van Boxtel, Zaalberg, Goudena, \& Matthys, 2006).

\section{AUTHOR NOTE}

Research was funded by a grant from the John Templeton Foundation and a fellowship from Eli Lilly and Company to S.D.P. The authors thank Victoria Laur, Eric Sjoberg, Judy Wan, Leah Abraham, Sarah Young, Nina Strohminger, and John Wang for help with the collection of data and Steven Wengrovitz for help in preparation of the original manuscript. David Meyer and John Jonides also provided helpful feedback on the task. Correspondence relating to this article should be addressed to S. D. Preston, 530 Church Street, Department of Psychology, University of Michigan, Ann Arbor, MI 48109 (e-mail: prestos@umich.edu).

Note-This article was accepted by the previous editorial team, when John Jonides was Editor.

\section{REFERENCES}

Adolphs, R., Damasio, H., Tranel, D., Cooper, G., \& Damasio, A. R. (2000). A role for somatosensory cortices in the visual recognition of emotion as revealed by three-dimensional lesion mapping. Journal of Neuroscience, 20, 2683-2690.

Algom, D., Chajut, E., \& Lev, S. (2004). A rational look at the emotional Stroop phenomenon: A generic slowdown, not a Stroop effect. Journal of Experimental Psychology: General, 133, 323-338.

Archer, J., Hay, D. C., \& Young, A. W. (1994). Movement, face processing, and schizophrenia: Evidence of a differential deficit in expression analysis. British Journal of Clinical Psychology, 33, 517-528.

BachmanN, T., \& Ort, M. (1992). Stroop-like interference in chess players' imagery: An unexplored possibility to be revealed by the adapted moving-spot task. Psychological Research, 54, 27-31.

Bargh, J. A., Chen, M., \& Burrows, L. (1996). Automaticity of social behavior: Direct effects of trait construct and stereotype activation on action. Journal of Personality \& Social Psychology, 71, 230-244.

Baron-Cohen, S., Ring, H., Moriarty, J., Schmitz, B., Costa, D., \& 
ELL, P. (1994). Recognition of mental state terms: Clinical findings in children with autism and a functional neuroimaging study of normal adults. British Journal of Psychiatry, 165, 640-649.

Beck, A. T., Steer, R. A., \& Garbin, M. G. (1988). Psychometric properties of the Beck Depression Inventory: Twenty-five years of evaluation. Clinical Psychology Review, 8, 77-100.

BUCK, R. (1980). Nonverbal behavior and the theory of emotion: The facial feedback hypothesis. Journal of Personality \& Social Psychology, 38, 811-824.

Burton, L. A., Wyatt, G., Rabin, L., Frohlich, J., Vardy, S. B., DimiTRI, D., \& LABAR, D. (2003). Perception and priming of affective faces in temporal lobectomy patients. Journal of Clinical \& Experimental Neuropsychology, 25, 348-360.

Carr, L., Iacoboni, M., Dubeau, M. C., Mazziotta, J. C., \& LENZI, G. L. (2003). Neural mechanisms of empathy in humans: A relay from neural systems for imitation to limbic areas. Proceedings of the National Academy of Sciences, 100, 5497-5502.

DARWIN, C. (1998). The expression of the emotions in man and animals (3rd ed.). New York: Oxford University Press. (Original work published 1872)

Decety, J., \& Chaminade, T. (2003). Neural correlates of feeling sympathy. Neuropsychologia, 41, 127-138.

De Wied, M., van Boxtel, A., Zaalberg, R., Goudena, P. P., \& MatTHYS, W. (2006). Facial EMG responses to dynamic facial expressions in boys with disruptive behavior disorders. Journal of Psychiatric Research, 40, 112-121.

Dimberg, U., Thunberg, M., \& Elmehed, K. (2000). Unconscious facial reactions to emotional facial expressions. Psychological Science, 11, 86-89.

DoherTy, R. W. (1997). The emotional contagion scale: A measure of individual differences. Journal of Nonverbal Behavior, 21, 131-154.

EkMAN, P. (1973). Darwin and cross-cultural studies of facial expression. In P. Ekman (Ed.), Darwin and facial expression: A century of research in review (pp. 1-83). New York: Academic Press.

Ekman, P. (2001). Telling lies: Clues to deceit in the marketplace, politics, and marriage. New York: Norton.

Ekman, P., \& Friesen, W. V. (1976). Pictures of facial affect [Slides]. Palo Alto, CA: Consulting Psychologists Press.

Ekman, P., Levenson, R. W., \& Friesen, W. V. (1983). Autonomic nervous system activity distinguishes among emotions. Science, 221, 1208-1210.

Ekman, P., Sorenson, E. R., \& Friesen, W. V. (1969). Pan-cultural elements in facial displays of emotion. Science, 164, 86-88.

Etkin, A., Egner, T., Peraza, D. M., Kandel, E. R., \& Hirsch, J. (2006). Resolving emotional conflict: A role for the rostral anterior cingulate cortex in modulating activity in the amygdala. Neuron, 51, 871-882.

Feyereisen, P., Verbeke-Dewitte, C., \& Seron, X. (1986). On fluency measures in aphasic speech. Journal of Clinical \& Experimental Neuropsychology, 8, 393-404.

Field, T., Pickens, J., Fox, N. A., Gonzalez, J., \& Nawrocki, T. (1998). Facial expression and EEG responses to happy and sad faces/ voices by 3-month-old infants of depressed mothers. British Journal of Developmental Psychology, 16, 485-494.

Galati, D., Scherer, K. R., \& Ricci-Bitti, P. E. (1997). Voluntary facial expression of emotion: Comparing congenitally blind with normally sighted encoders. Journal of Personality \& Social Psychology, 73, 1363-1379.

Grafton, S. T., Fadiga, L., Arbib, M. A., \& Rizzolatti, G. (1997). Premotor cortex activation during observation and naming of familiar tools. NeuroImage, 6, 231-236.

Gray, J. M., Young, A. W., Barker, W. A., Curtis, A., \& Gibson, D. (1997). Impaired recognition of disgust in Huntington's disease gene carriers. Brain, 120, 2029-2038.

Haas, B. W., Omura, K., Constable, R. T., \& Canli, T. (2006). Interference produced by emotional conflict associated with anterior cingulate activation. Cognitive, Affective, \& Behavioral Neuroscience, 6, 152-156.

Hess, U., Blairy, S., \& KLECK, R. E. (1997). The intensity of emotional facial expressions and decoding accuracy. Journal of Nonverbal Behavior, 21, 241-257.

Hobson, R. P., Ouston, J., \& LeE, A. (1989). Naming emotion in faces and voices: Abilities and disabilities in autism and mental retardation. British Journal of Developmental Psychology, 7, 237-250.

Hommel, B. (2003). Planning and representing intentional action. The Scientific World Journal, 3, 593-608.

IZARD, C. E. (1994). Innate and universal facial expressions: Evidence from developmental and cross-cultural research. Psychological Bulletin, 115, 288-299.

Kamio, Y., Wolf, J., \& Fein, D. (2006). Automatic processing of emotional faces in high-functioning pervasive developmental disorders: An affective priming study. Journal of Autism \& Developmental Disorders, 36, 155-167.

Lanzetta, J. T., Cartwright-Smith, J., \& Kleck, R. E. (1976). Effects of nonverbal dissimulation on emotional experience and autonomic arousal. Journal of Personality \& Social Psychology, 33, 354-370.

Levenson, R. W., Ekman, P., Heider, K., \& Friesen, W. V. (1992). Emotion and autonomic nervous system activity in the Minangkabau of West Sumatra. Journal of Personality \& Social Psychology, 62, 972-988.

MAcLeOD, C. M. (1991). Half a century of research on the Stroop effect: An integrative review. Psychological Bulletin, 109, 163-203.

McKenna, F. P., \& Sharma, D. (1995). Intrusive cognitions: An investigation of the emotional Stroop task. Journal of Experimental Psychology: Learning, Memory, \& Cognition, 21, 1595-1607.

Murphy, S. T., \& ZAJONC, R. B. (1993). Affect, cognition, and awareness: Affective priming with optimal and suboptimal stimulus exposures. Journal of Personality \& Social Psychology, 64, 723-739.

Niedenthal, P. M. (1990). Implicit perception of affective information. Journal of Experimental Social Psychology, 26, 505-527.

Palermo, R., \& Coltheart, M. (2004). Photographs of facial expression: Accuracy, response times, and ratings of intensity. Behavior Research Methods, Instruments, \& Computers, 36, 634-638.

Pessoa, L., Japee, S., Sturman, D., \& Ungerleider, L. G. (2006). Target visibility and visual awareness modulate amygdala responses to fearful faces. Cerebral Cortex, 16, 366-375.

Pessoa, L., Padmala, S., \& Morland, T. (2005). Fate of unattended fearful faces in the amygdala is determined by both attentional resources and cognitive modulation. NeuroImage, 28, 249-255.

Phillips, M. L., Williams, L. M., Heining, M., Herba, C. M., RusSELL, T., ANDREW, C., ET AL. (2004). Differential neural responses to overt and covert presentations of facial expressions of fear and disgust. NeuroImage, 21, 1484-1496.

Preston, S. D., Bechara, A., Damasio, H., Grabowski, T. J., StansField, R. B., Mehta, S., \& Damasio, A. R. (2007). The neural substrates of cognitive empathy. Social Neuroscience, 2, 254-275.

Preston, S. D., \& DE WAAL, F. B. M. (2002). Empathy: Its ultimate and proximate bases. Behavioral \& Brain Sciences, 25, 1-71.

Preston, S. D., \& Stansfield, R. B. (2006, April). Automatic processing of emotion in facial expressions: A true emotional Stroop effect. Paper presented at the annual meeting of the Cognitive Neuroscience Society, San Francisco.

Preston, S. D., \& Stansfield, R. B. (2007, May). The Emostroop effect: Task-irrelevant facial emotions are processed spontaneously, rapidly, and at the level of the specific emotion. Paper presented at the Neural Systems of Social Behavior Conference, Austin, TX.

Schneider, F., Gur, R. C., Koch, K., Backes, V., Amunts, K., Shah, N. J., ET AL. (2006). Impairment in the specificity of emotion processing in schizophrenia. American Journal of Psychiatry, 163, 442-447.

Schwarz, N., \& Clore, G. L. (1996). Feelings and phenomenal experiences. In E. T. Higgins \& A. W. Kruglanski (Eds.), Social psychology: Handbook of basic principles (pp. 433-465). New York: Guilford.

Shaver, P., Schwartz, J., Kirson, D., \& O'Connor, C. (1987). Emotion knowledge: Further exploration of a prototype approach. Journal of Personality \& Social Psychology, 52, 1061-1086.

Silver, H., Shlomo, N., Turner, T., \& Gur, R. C. (2002). Perception of happy and sad facial expressions in chronic schizophrenia: Evidence for two evaluative systems. Schizophrenia Research, 55, 171-177.

Singer, T., Seymour, B., O'Doherty, J., Kaube, H., Dolan, R. J., \& FRITH, C. D. (2004). Empathy for pain involves the affective but not sensory components of pain. Science, 303, 1157-1162.

Sprengelmeyer, R., Young, A. W., Calder, A. J., Karnat, A., LANGe, H., HÖMBERG, V., ET AL. (1996). Loss of disgust: Perception of faces and emotions in Huntington's disease. Brain, 119, 1647-1665. 
Sprengelmeyer, R., Young, A. W., Sprengelmeyer, A., Calder, A. J., Rowland, D., Perrett, D., et AL. (1997). Recognition of facial expressions: Selective impairment of specific emotions in Huntington's disease. Cognitive Neuropsychology, 14, 839-879.

Stapel, D. A., Koomen, W., \& RuYs, K. I. (2002). The effects of diffuse and distinct affect. Journal of Personality \& Social Psychology, 83, 60-74.

STroop, J. R. (1935). Factors affecting speed in serial verbal reactions. Journal of Experimental Psychology, 18, 643-662.

Thomas, K. M., Drevets, W. C., Whalen, P. J., Eccard, C. H., Dahl, R. E., Ryan, N. D., \& CASEY, B. J. (2001). Amygdala response to facial expressions in children and adults. Biological Psychiatry, 49, 309-316.
Wicker, B., Perrett, D. I., Baron-Cohen, S., \& Decety, J. (2003). Being the target of another's emotion: A PET study. Neuropsychologia, 41, 139-146.

Williams, J. M. G., Mathews, A., \& Macleod, C. (1996). The emotional Stroop task and psychopathology. Psychological Bulletin, 120, 3-24.

Winkielman, P., Berridge, K. C., \& Wilbarger, J. L. (2005). Unconscious affective reactions to masked happy versus angry faces influence consumption behavior and judgments of value. Personality \& Social Psychology Bulletin, 31, 121-135.

(Manuscript received May 16, 2007; revision accepted for publication November 14, 2007.) 